\title{
UNA NOTA SOBRE LA EVOLUCIÓN DE LA ESTRUCTURA DE INGRESOS Y GASTOS BANCARIOS: 1966-1979
}

\author{
ALAIN IZE*
}

El Colegio de México

\section{Estabilidad y crisis en la intermediación financiera}

Si SE OBSERVA la evolución, en pesos constantes, de la captación y del crédito total para la banca privada y mixta a lo largo del período 66-79, se notan claramente (véase la gráfica 1) dos subperíodos con características radicalmente distintas. En el primer subperíodo, que abarca desde 66 hasta 71, tanto la captación como el crédito crecieron en forma uniforme, mientras que sufrieron fuertes variaciones y tendieron a estancarse en el periodo posterior. ${ }^{1}$ Esta clara separación es fácilmente explicable por las características del crecimiento económico en cada uno de estos períodos: mientras que 66-71 fue un período de crecimiento estable sin inflación, 72-79 fue un período de crisis con altas tasas de inflación, incertidumbre cambiaria y tasas de crecimiento muy variables.

El objetivo de esta nota es de comparar el comportamiento de los bancos en ambos subperíodos y de examinar en particular cómo ajustaron sus ingresos y gastos para hacer frente a la crisis financiera de los setentas. El análisis aporta algunas indicaciones interesantes sobre el funcionamiento de la industria bancaria.

* Este trabajo forma parte de un proyecto conjunto El Colegio de México-Banco de México. El autor agradece la asistencia de Nisso Bucay en la elaboración del trabajo y la ayuda proporcionada por Manuel Basurto, Armando Baqueiro y Gabriel Vera del Banco de México, en la obtención y análisis de la información.

1 El crédito tendió a estancarse antes que la captación (se observa este estancamiento desde 71), debido a la existencia de fuertes excesos de oferta en el mercado crediticio en los años 71-72. Para una más amplia documentación de este fenómeno véase Calderón, Cárdenas e Ize (1980). 


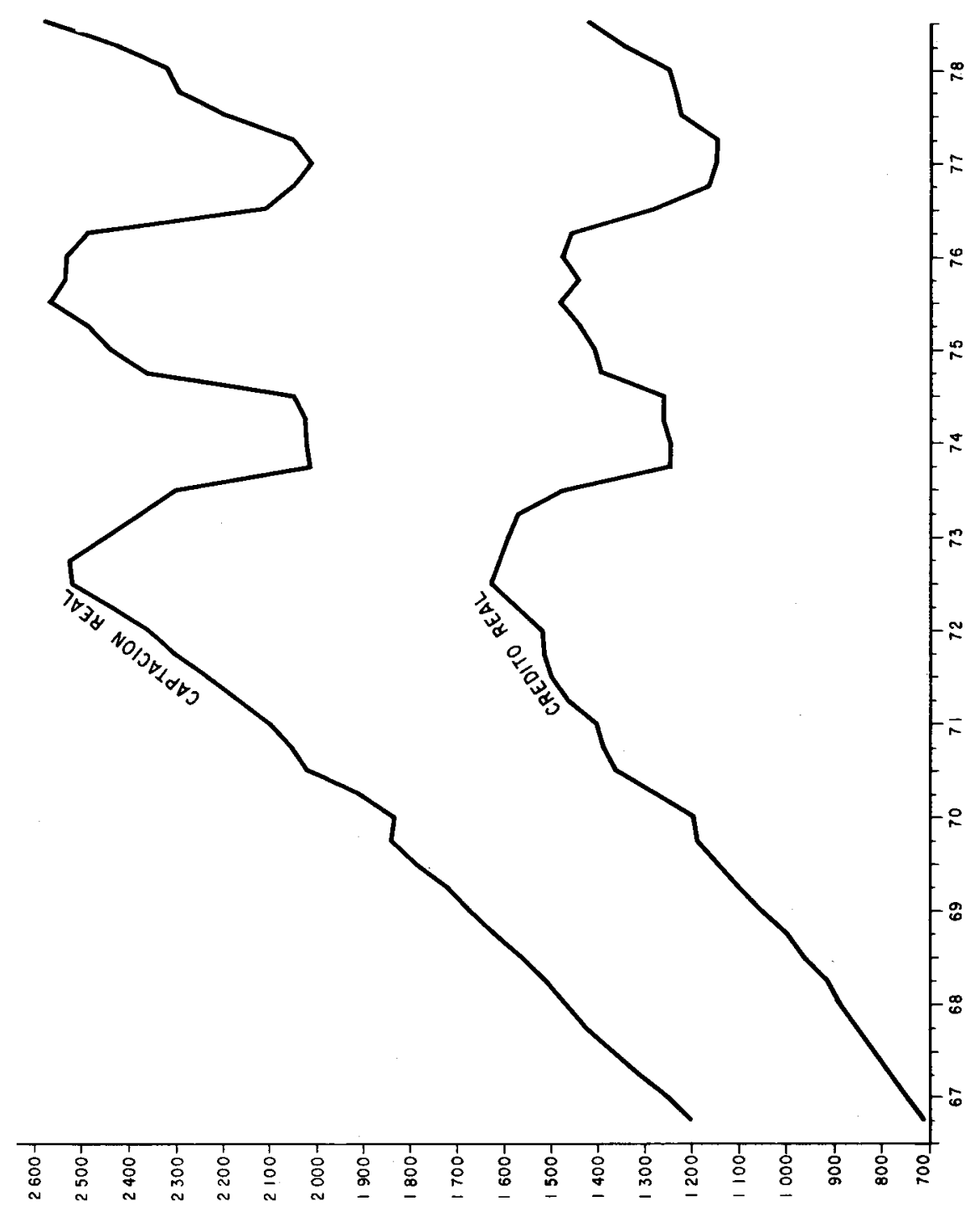




\section{Análisis del estado de resultados}

Un primer análisis de la evolución de gastos e ingresos bancarios puede ser llevado a cabo a partir del estado de resultados, escrito en la forma siguiente:

$$
\mathrm{rL}+\mathrm{IR}+\mathrm{OI}-\mathrm{GF}-\mathrm{GT}(\mathrm{W} / \mathrm{P})-\mathrm{GA}=\mathrm{T}
$$

en donde $\mathrm{r}$ es la tasa activa promedio, $\mathrm{L}$ es el crédito otorgado en el período, IR corresponde a los ingresos derivados de las reservas depositadas en el Banco de México, OI son otros ingresos (comisiones y situaciones cobradas, recuperaciones, cambios, dividendos, reportes, etc.), GF son gastos financieros por pagos de intereses, GT son gastos en trabajo (sueldos y sobresueldos, gratificaciones, prestaciones, etc.), GA son gastos administrativos (rentas, papelería, publicidad, seguros, mantenimiento, castigos, etc.) y $\mathrm{T}$ son las utilidades brutas del periodo. L, IR, OI, GF, GA y T están expresadas en términos reales, dividiendo las magnitudes nominales por el índice de precios. GT está también en términos reales, pero está dividido por el índice de salarios, lo que es más apropiado en este caso; el término en GT tiene por lo tanto que ser multiplicado por el salario real, W/P, para que se respete la identidad contable.

Dado que el Banco de México es el que fija la tasa pasiva, los gastos financieros están dados exógenamente, lo mismo que los ingresos por reservas, el crédito disponible ${ }^{2}$ y los otros ingresos. Por otra parte, la tasa activa, los gastos en trabajo, los gastos administrativos y las utilidades son variables que están bajo el control directo de los bancos. $^{3}$ Es preciso examinar en qué forma los bancos han ajustado cada una de estas cuatro variables para hacer frente a la fuerte reducción en sus recursos que experimentaron en los setentas.

Tomando como base el período 67-71, se extrapolaron ${ }^{4}$ los valores de tendencias para el período 72-79. Estos valores, junto con los

2 Los bancos pueden depositar reservas en exceso en el Banco de México y tienen por lo tanto cierto control sobre la oferta de crédito. Sin embargo estos excesos de reserva tienen magnitudes relativamente pequeñas en comparación con los órdenes de magnitud que consideremos aquí y no serán por lo tanto tomados explícitamente en cuenta.

3 Es importante sin embargo notar que si la oferta de fondos prestables está dada y si la tasa activa tuviese que equilibrar constantemente el mercado de crédito, esta última variable cesaría de ser una variable bajo el control estricto de los bancos. Pero parecen existir ciertas indicaciones que el mercado de fondos prestables se encuentra frecuentemente en desequilibrio; (para un análisis de este punto véase Calderón, Cárdenas e Ize (1980) ).

4 El método usado para estas extrapolaciones fue una regresión sencilla sobre los logaritmos de las variables. 
valores históricos de las cuatro variables endógenas (r, GT, GA y T) aparecen en las gráficas 2 a 5 . Se nota inmediatamente que tanto los gastos en trabajo y los gastos administrativos, como las utilidades se ajustaron en baja en los años 72-79, en relación con su tendencia histórica en los años 67-71..$^{5}$ La tasa activa se ajustó en alza, haciendo aumentar los ingresos financieros, como era también de esperarse. Se nota también que ambos tipos de gasto tienden a acercarse otra vez de sus valores de tendencia en 1979; este fenómeno es aún más sensible para las utilidades que prácticamente recuperan su valor de tendencia en 79.

Para evaluar mejor estos ajustes desde un punto de vista cuantitativo se calcularon en primer lugar los promedios de desviación, sobre el período $72-79$, de las cuatro variables endógenas con respecto a su tendencia, alcanzándose los resultados siguientes: ${ }^{6}$

Cuadro 1

PROMEDIOS DE DESVIACIÓN

\begin{tabular}{cccc}
\hline Tasa activa & Utilidades & $\begin{array}{c}\text { Gastos en } \\
\text { trabajo }\end{array}$ & $\begin{array}{c}\text { Gastos } \\
\text { Administrativos }\end{array}$ \\
\hline$-15.36 \%$ & $21.75 \%$ & 11.938 & 53.608
\end{tabular}

Estos resultados parecen indicar que la variable que los bancos ajustaron más fácilmente fue la de gastos administrativos, ya que éstos se redujeron más de un $50 \%$ en promedio en el período $72-79$. En cambio, la variable que muestra más rigidez de ajuste es la de gastos en trabajo, que sólo fue ajustada un $12 \%$ en promedio. Las utilidades ocupan un término intermedio con un $22 \%$ de ajuste. La tasa activa tiene un promedio de ajuste relativamente pequeño $(15 \%)$; sin embargo, este ajuste fue ampliándose en todo el periodo 72-79, hasta alcanzar cerca de un $40 \%$ en 79 . Esto parece indicar que ha existido

5 Las tasas reales de crecimiento anual promedio entre 66 y 71 fueron de $16.77 \%$, 8.9690 y 9.5890 respectivamente, para los gastos administrativos, gastos en trabajo y utilidades.

6 Se tomó el promedio aritmético de la diferencia entre el valor de tendencia y el valor histórico sobre el valor de tendencia. 
LN. Utilidades totales

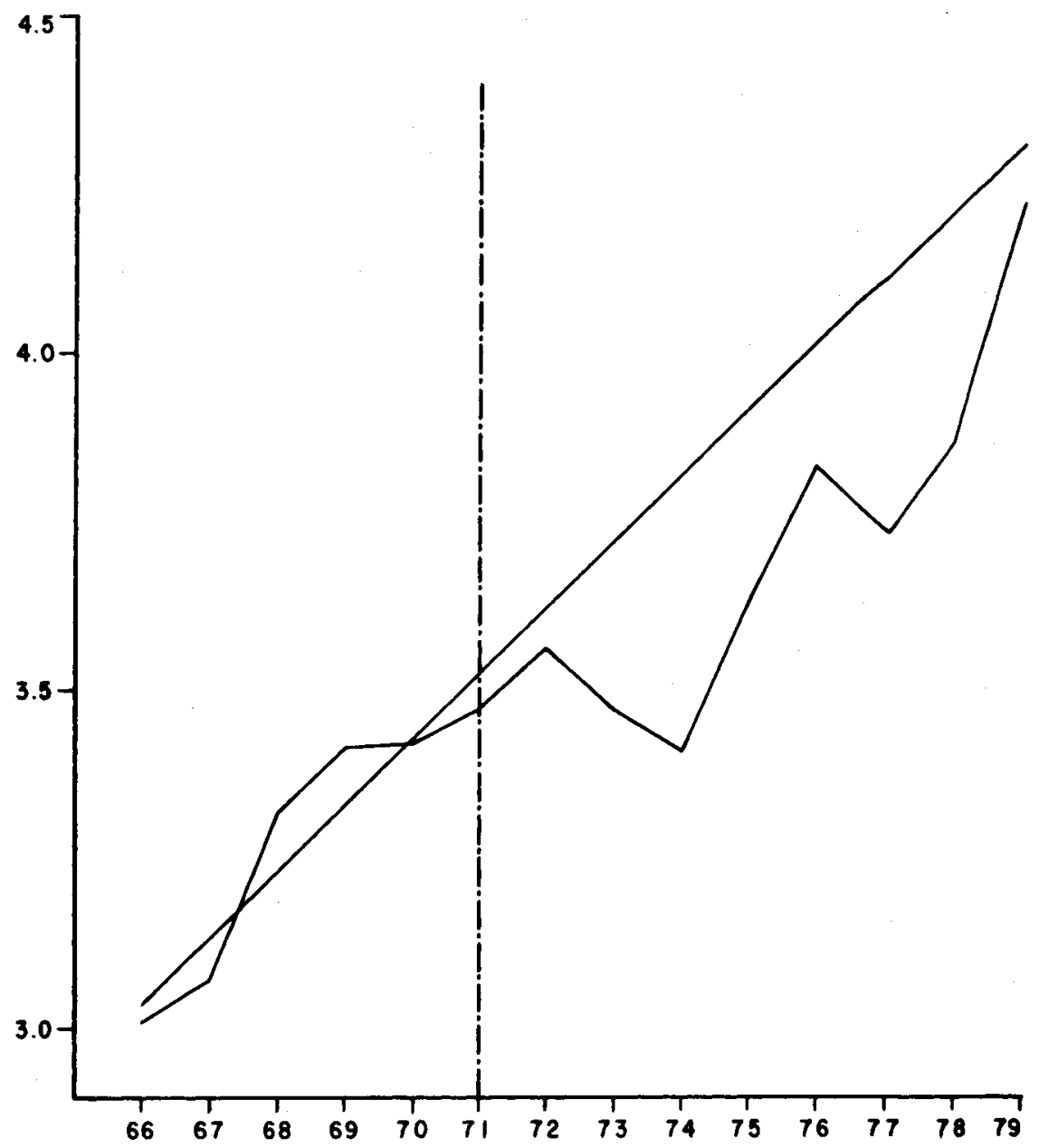

un fuerte rezago en el ajuste de la tasa activa. Por otra parte, es claro que los bancos no pudieron (o no quisieron) hacer caer todo el peso del ajuste sobre la tasa de interés, de tal forma que se mantuvieron constantes sus gastos y sus utilidades.

Los resultados obtenidos anteriormente no nos permiten sin embargo apreciar cuáles variables absorbieron la mayor parte del ajuste to- 
Gráfica 3

LN. Gastos Administrativos

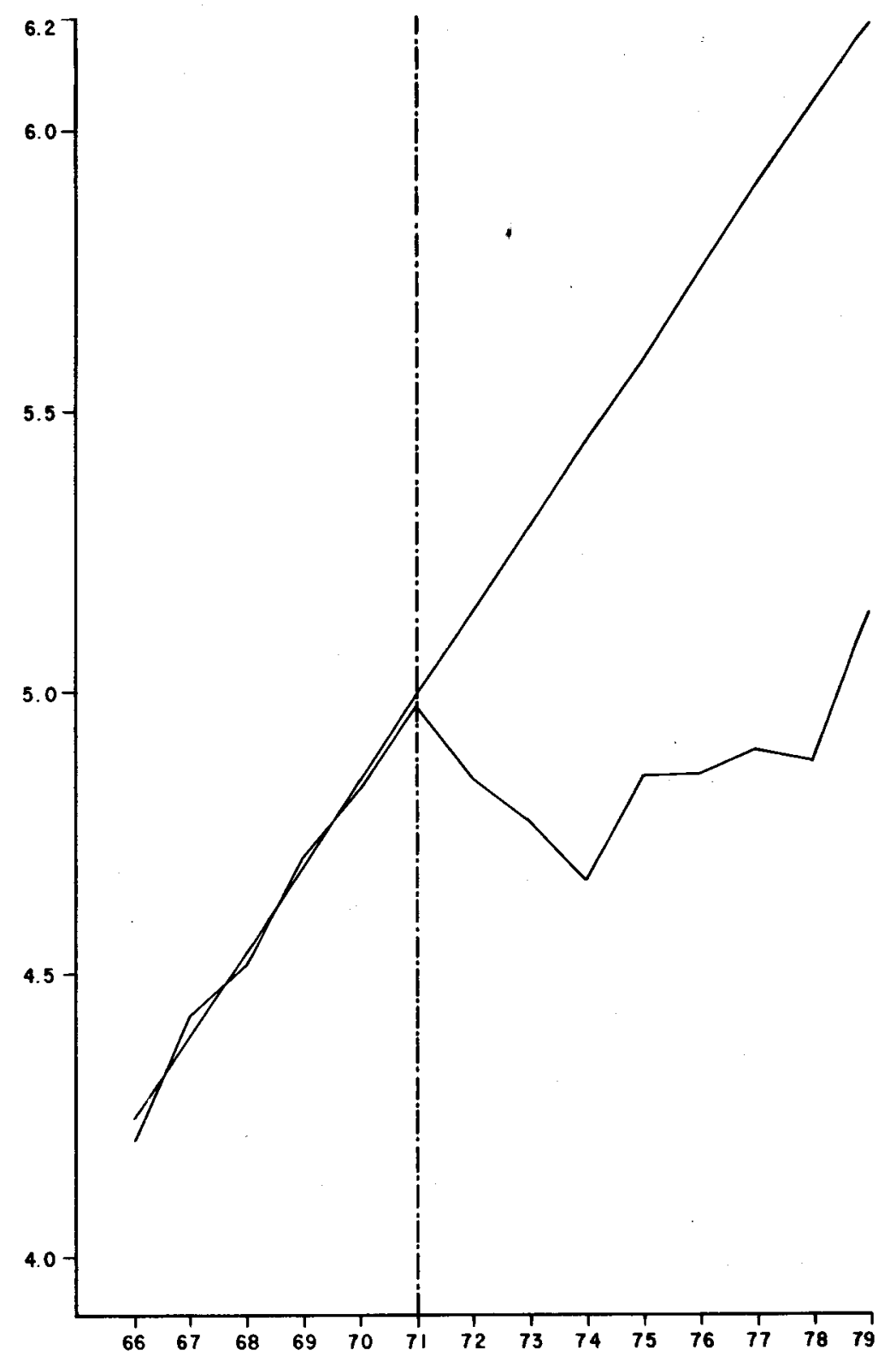




\section{Gráfica 4}

LN. Gastos en trabajo

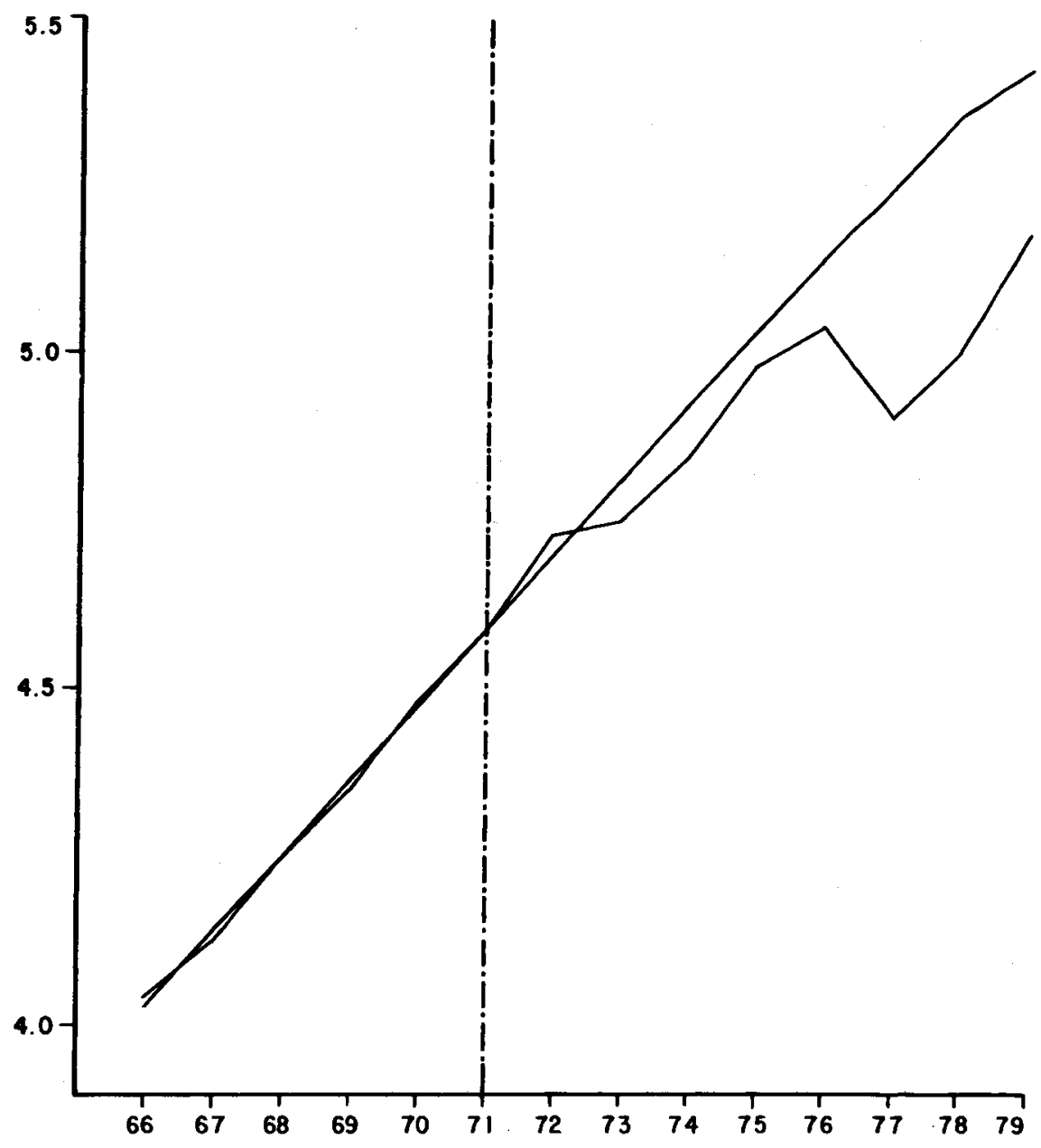

tal que se requería para hacer frente a la reducción en la captación. Para tal efecto, se calcularon, a partir'de la ecuación 1 , valores de ajuste para cada una de las cuatro variables endógenas, suponiendo que las otras tres variables endógenas siguieran sus valores de tendencia y tomando los valores históricos para las variables exógenas. Se derivaron después índices anuales de ajuste como la diferencia entre valores his- 


\section{Gráfica 5}

Tasa Activa

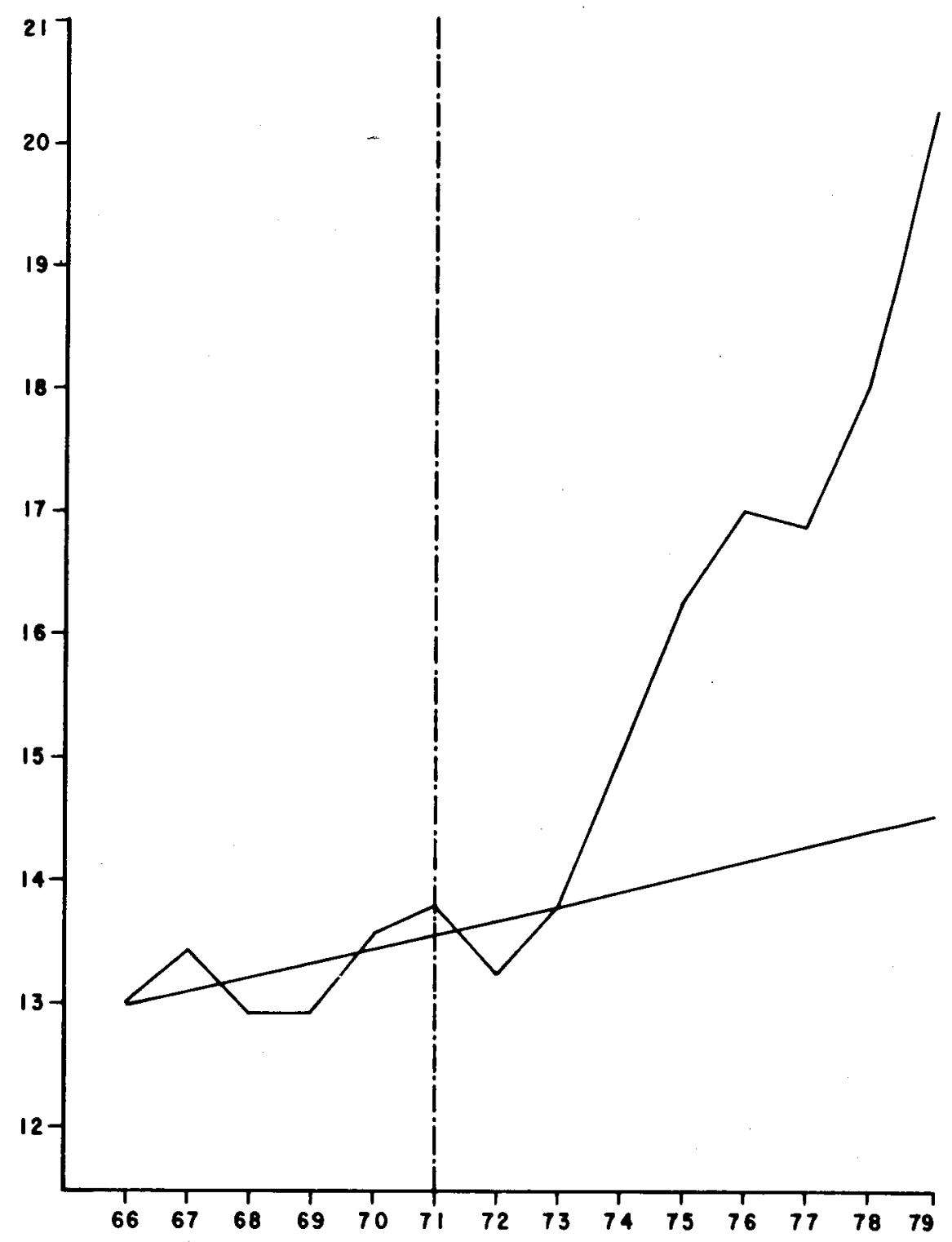


tóricos y de tendencia sobre la diferencia entre valores ajustados y de tendencia, de tal forma que este índice valiera uno en caso de que la variable correspondiente haya absorbido todo el peso del ajuste en el año considerado, y cero en caso de que no haya absorbido ninguna parte del ajuste. Finalmente, se calcularon índices ponderados promedio para el período $72-79$, los coeficientes de ponderación siendo proporcionales a la participación del ajuste anual dentro del ajuste total. ${ }^{7}$ Los resultados obtenidos aparecen en el siguiente cuadro.

Cuadro 2

COEFICIENTES DE AJUSTE TOTAL

\begin{tabular}{cccc}
\hline Tasa activa & Utilidades & $\begin{array}{c}\text { Gastos en } \\
\text { trabajo }\end{array}$ & $\begin{array}{c}\text { Gastos } \\
\text { Administrativos }\end{array}$ \\
\hline 22.8 & 4.38 & 8.3 & 63.9 \\
\hline
\end{tabular}

Por otra parte, si en vez de utilidades puras, se toma como variable de control la relación de utilidades a captación y se repite el mismo procedimiento, se obtienen los resultados del cuadro 3 .

Cuadro 3

COEFICIENTES DE AJUSTE CON UTILIDADES Y CAPTACIONES

\begin{tabular}{cccc}
\hline Tasa activa & Utilidades & $\begin{array}{c}\text { Gastos en } \\
\text { trabajo }\end{array}$ & $\begin{array}{c}\text { Gastos } \\
\text { Administrativos }\end{array}$ \\
\hline 24.68 & -4.98 & 7.68 & 70.48
\end{tabular}

El ajuste de la variable de utilidades sobre captación es ahora negativo.

De estos resultados se desprende lo siguiente:

a) La variable de gastos administrativos es la que ha soportado la gran mayoría del ajuste (alrededor de las dos terceras partes).

b) Dado el signo positivo del ajuste de utilidades puras y el signo negativo del ajuste de utilidades sobre captación, y dada la relativa pequeñez de ambos ajustes, se puede quizás inferir que los bancos han tratado de mantener un compromiso entre ambos tipos de objetivos, el de unas utilidades estables y el de una relación de utilidades

7 Estos cálculos son expuestos más formalmente en el apéndice. 
a captación también estable. ${ }^{8}$ Todo parece así señalar que un objetivo prioritario de la industria bancaria en esta época de crisis fue de no afectar sus utilidades, probablemente porque esto es un requerimiento indispensable para la imagen de la institución y la confianza que inspira.

c) Los gastos en trabajo han seguido una dinámica de crecimiento casi autónoma y no han soportado más que una parte muy pequeña del ajuste necesario, mientras que la tasa activa es la variable que más se ha ajustado después de la de gastos administrativos.

\section{Gastos administrativos}

Sería interesante analizar más en detalle el rubro de los gastos administrativos para ver cuáles han sido los renglones más dinámicos, o sea los que se han ajustado más en respuesta a la crisis financiera de los setentas. Desgraciadamente, el desglose necesario de la información no está disponible sino a partir de 1974 solamente. Lo que se puede, sin embargo, hacer es comparar las tasas de crecimiento de estos diferentes renglones entre 74 y 79. Dado que esta época fue de crecimiento sostenido para el total de los gastos administrativos (320\% sobre todo el período), los rubros que hayan crecido mucho más rápidamente que el promedio pueden interpretarse como los más dinámicos. Estas tasas de crecimiento, junto con la participación de cada rubro dentro del total de gastos administrativos, aparecen en el cuadro 4.

Los rubros importantes que crecieron mucho más rápidamente que el promedio son los de rentas pagadas (540\%), publicidad (520\%), correo, telégrafos y teléfonos $(425 \%)$, distribución interna de gastos $(389 \%)$ y otros gastos no especificados (380\%). Dos de estos rubros, el de correo, teléfonos y telégrafos y el de distribución de gastos están asociados en cierta forma al crecimiento de las sucursales; el primero, porque más sucursales implican más gastos de comunicación y el segundo porque corresponde a gastos propios de las sucursales. Es por lo tanto necesario tomar en cuenta que la cantidad de sucursales crecieron por un $40 \%$ aproximadamente en este período, lo que quizás justifique que los gastos que les estén asociados hayan crecido más que el promedio. El crecimiento muy fuerte de gastos en publicidad también puede deberse a una mayor competencia entre bancos debido al mayor número de sucursales y al estancamiento de la captación.

8 Dada la fuerte correlación existente entre captación y capital contable de los bancos, la relación de utilidades a captación puede interpretarse como un ínđice de rendimiento del capital invertido. 
Quedan entonces dos rubros por explicar, el de rentas pagadas y el de gastos no especificados; el primero puede estar asociado con el aumento de sucursales; pero esto no permitiría justificar más que una parte pequeña del aumento total. Ambos rubros tienen en realidad una característica en común que quizás explique mejor su rápido crecimiento: pueden ser alterados con relativa facilidad por los bancos. ${ }^{9}$

Si se toma además en cuenta que las tasas reales anuales de crecimiento de los gastos administrativos en los dos subperíodos de "mejoría", el de 66-71 y el de 74-79, fueron respectivamente de $16.8 \%$ y $9.9 \%$, cifras muy superiores a las tasas de crecimiento de la captación real, los gastos en trabajo, sucursales y utilidades, se percibe cierta asociación entre ingresos y gastos "volátiles": variaciones fuertes en el ingreso "disponible" de los bancos parecen repercutir en amplias variaciones de los gastos más fácilmente ajustables; estos gastos crecen muy rápidamente en tiempos de "buenos" negocios y decaen también brutalmente en tiempos más difíciles. Parece existir así una relación estrecha entre utilidades "ex-ante" y gastos.

\section{Sucursales y empleo}

Si examinamos ahora la evolución de las sucursales bancarias a lo largo del período 66-76, se observa (véase la gráfica 6) que su crecimiento ha sido prácticamente constante. El promedio de desviación del número de sucursales en el subperíodo $72-76$ con respecto a su tendencia de crecimiento (de 6.9\% anual) en el subperíodo 66-71 es de $3.02 \%$, cifra mucho más baja que cualquiera de las que habían sido encontradas para las demás variables. Por otra parte, se aprecia claramente en la gráfica 7 que la captación (en términos reales) por sucursal cayó drásticamente a partir de 73 recuperándose sólo levemente en 78 y 79 . Finalmente, si se grafica la cifra de gastos en trabajo (deflacionados por el índice de salarios) sobre el número de sucursales (gráfica 8) se observa que después de un crecimiento constante entre 67 y 72 , esta cifra cayó de 73 a 76.

Estos resultados reflejan dos puntos interesantes:

a) A pesar de una fuerte caida en la captación, el crecimiento de las sucursales siguió una dinámica prácticamente autónoma.

9 Las rentas pagadas son en su mayoría rentas imputadas que fijan los propios bancos sin seguir lineamientos muy estrictos. 
Cuadro 4

TASAS DE CRECIMIENTO Y PESO RELATIVO DE LOS RENGLONES DE GASTO ADMINISTRATIVO

\begin{tabular}{lcc}
\hline Renglones & $\begin{array}{c}\text { Tasa de } \\
\text { crecimiento }\end{array}$ & $\begin{array}{c}\text { Participacion dentro } \\
\text { del total de gastos } \\
\text { administrativos en } 79\end{array}$ \\
\hline Publicidad & 520 & 4.4 \\
Rentas pagadas por locales & 540 & 10.4 \\
Reparación de equipo & 353 & 3.4 \\
Distribución interna de gastos & 389 & 5.4 \\
Gastos no especificados & 380 & 14.2 \\
Ajustes de reservas para pensiones & 225 & 6.4 \\
Seguros & 290 & 2.1 \\
Utiles y gastos de aseo & 417 & 2.5 \\
Correo, telegrafos, telefonos & 425 & 4.8 \\
Papelerla & 340 & 8.0 \\
Luz, agua y calefaccion & 292 & 1.8 \\
Mensajeria & 331 & 2.1 \\
Comisiones y sitiaciones pagadas & 348 & 5.7 \\
Castigos y depreciaciones & 218 & 13.2 \\
Varios & - & 15.3 \\
\hline
\end{tabular}

b) Los bancos, a raíz de la crisis financiera de los setentas, parecen haber hecho ciertos esfuerzos por mejorar su eficiencia en el uso del factor trabajo.

\section{Minimización de costos y dinámica de crecimiento}

El análisis llevado a cabo en este estudio parece indicar un tipo de comportamiento bastante peculiar por parte de los bancos y que podría ser esquematizado en la siguiente forma:

a) En el corto plazo el ingreso neto disponible de los bancos está dado. Se obtiene deduciendo los gastos financieros y los gastos en trabajo (ambos prácticamente fijos en el corto plazo) de los ingresos totales, en su mayoría ingresos financieros dados por las con- 


\section{Gráfica 6}

Sucursales

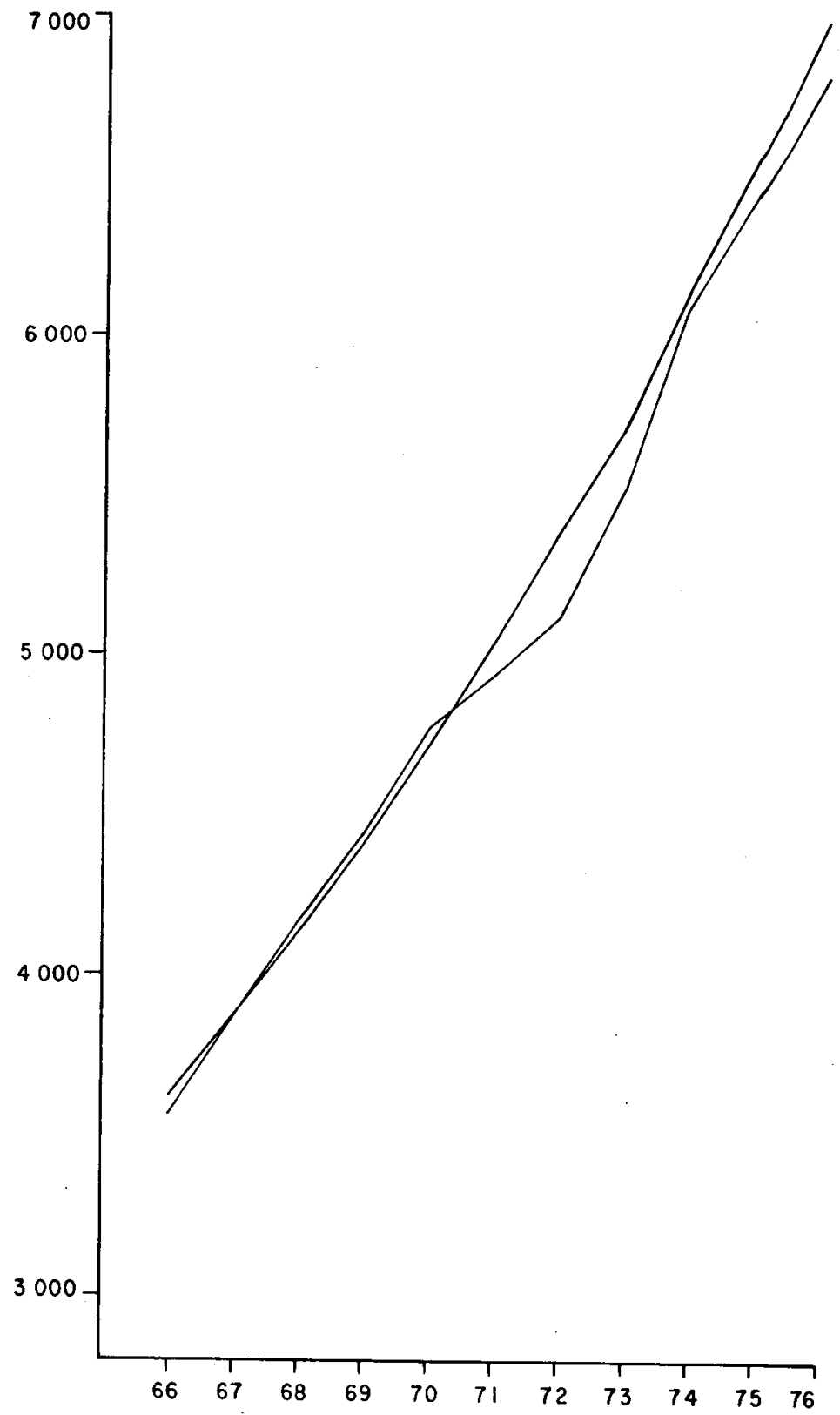




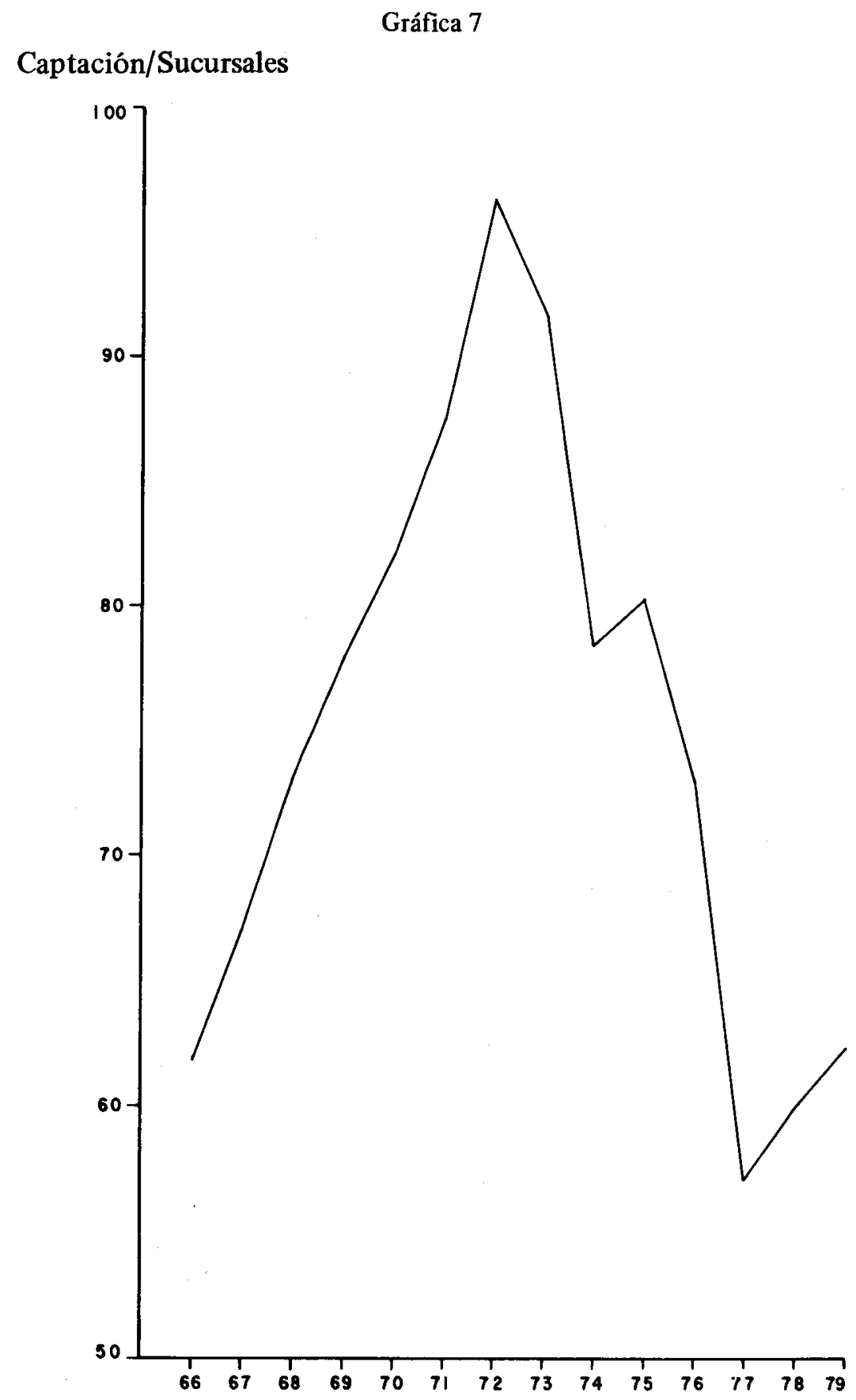


Gráfica 8

Empleo/Sucursales

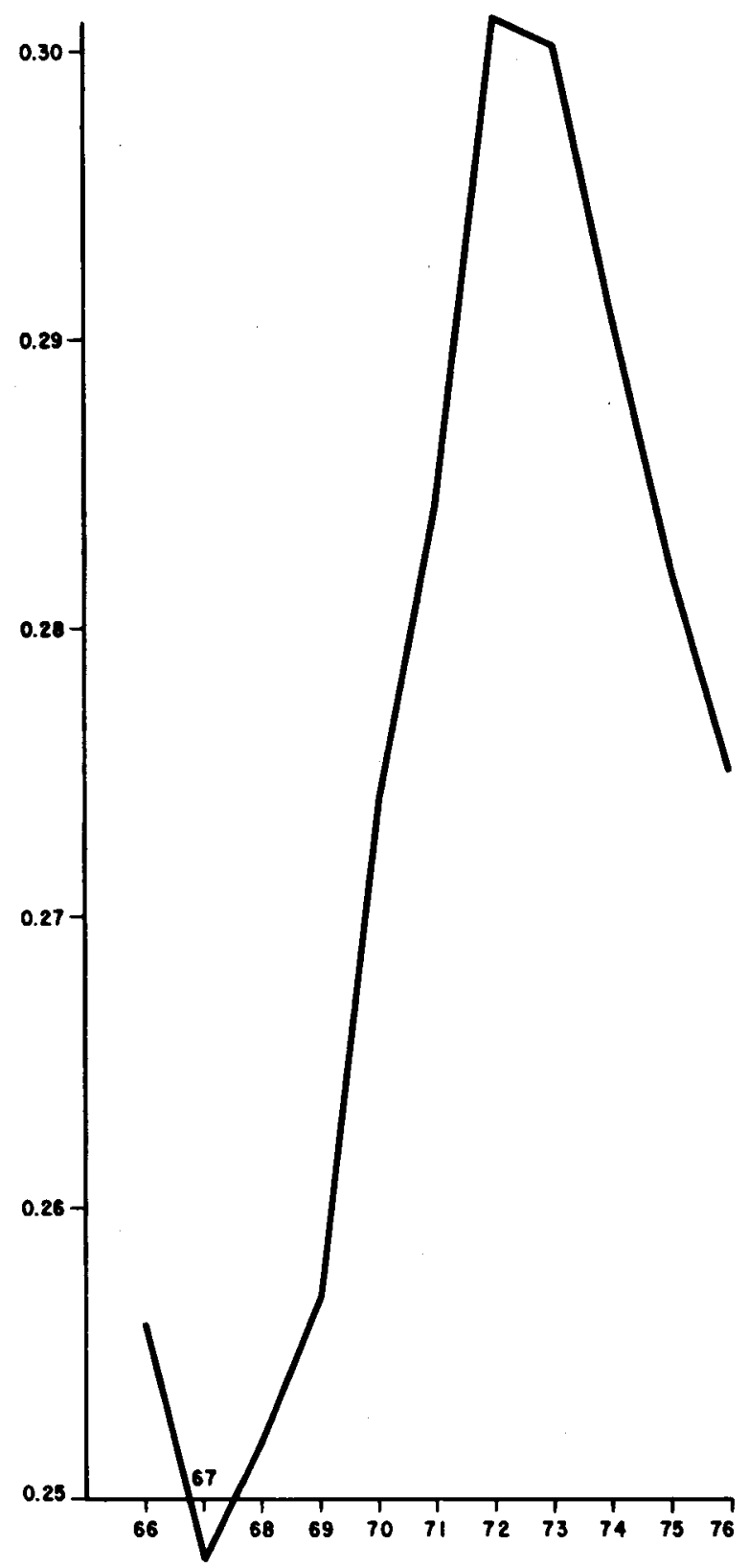


diciones de mercado. ${ }^{10}$ El gran diferencial que existe entre tasas activas y tasas pasivas de interés asegura que este ingreso neto disponible sea lo suficientemente alto para cubrir ampliamente los gastos administrativos indispensables.

b) El ingreso remanente puede entonces ser retenido como utilidades o "gastado" en renglones no esenciales y de fácil ajuste. El objetivo que parecen perseguir los bancos en el corto plazo es de mantener cierto ritmo de crecimiento de las utilidades que constituya un compromiso entre una "tasa de ganancia" constante y un ritmo de crecimiento de las utilidades puras constantes. Tras satisfacer este objetivo de ganancias, la variable residual que se ajusta para hacer cuadrar la cuenta de ingresos y gastos es la de gastos administrativos, y dentro de esta cuenta los renglones más "volátiles" son, por ejemplo, las rentas imputadas y los gastos diversos no especificados. Este mecanismo, de ser cierto, se contrapondría radicalmente al principio neoclásico usual de maximización de ganancias-minimización de costos.

c) En el más largo plazo, los bancos parecen tener un segundo objetivo que es el de asegurar una mayor participación dentro de la industria, o por lo menos el de no perder su participación relativa. Con este fin abren sucursales, aumentan su demanda de empleo y sus gastos en publicidad. Dado los niveles de rendimiento "exante" que consiguen, los bancos disponen de amplios márgenes para elevar el número de sus sucursales a pesar de que la captación por sucursal se reduzca drásticamente. Es probable que este crecimiento de sucursales no sea óptimo para el sistema financiero en general, pero los bancos, individualmente, se ven obligados a ello para no perder terreno frente a sus competidores. Es interesante notar, por otra parte, que la dinámica de crecimiento de las sucursales parece ser totalmente autónoma, ya sea porque se establece una especie de modus vivendi (o modus crescendi) entre bancos o porque el Banco de México regula este crecimiento. Es necesario, sin embargo, hacer resaltar que a raiz de la crisis financiera de los setentas, los bancos redujeron en cierta medida el ritmo de crecimiento de sus gastos de trabajo, lo que parece reflejar

10 Es importante hacer notar que en los setentas los bancos no han ajustado la tasa activa lo suficiente para lograr mantener un nivel de ingreso neto disponible constante. Esto puede reflejar restricciones de equilibrio del mercado crediticio (la tasa activa no pudo ser elevada más allá de su nivel de equilibrio); pero quizás también refleje restricciones más amplias de orden político ya que tasas más altas podrían haber dañado la imagen de la industria. 
una mayor preocupación por mejorar su eficiencia en el uso de este factor.

\section{Conclusiones}

Este trabajo sugiere que los mecanismos de comportamiento de la industria bancaria en México difieren ampliamente de los mecanismos neoclásicos usuales. En el corto plazo, el objetivo perseguido por los bancos parece ser el de mantener un nivel deseado de ganancias mediante un ajuste residual de sus costos, en vez de un objetivo de maximización de ganancias-minimización de costos. Esto plantea serios problemas de eficiencia en el uso de los recursos canalizados hacia los bancos. ${ }^{11}$ Lleva, por otra parte, a cuestionarse sobre la razón de ser de este objetivo de ganancias deseadas. Una posible respuesta es de orden político ya que unas ganancias demasiado altas afectarian negativamente la imagen de la industria; por otra parte, ganancias demasiado bajas dañarían también la imagen de solidez y de confianza que pueden tener los accionistas y el público en general sobre cada banco en particular. Pero otra posible respuesta a este objetivo de ganancias no excesivas es de orden fiscal, por razones obvias.

Este estudio sugiere también que en el más largo plazo el objetivo perseguido por los bancos es de aumentar, o por lo menos de no reducir, su participación dentro de la industria. Esto induce un crecimiento constante y casi autónomo de las sucursales, independiente de las condiciones de mercado, y en particular del hecho que puede reducirse mucho la captación real por sucursal. Esta situación podría desembocar eventualmente, si no es que existe ya cusde ailora, en un serio desperdicio de recursos, al existir mucho más sucursales que las que son socialmente deseables.

El origen de ambos tipos de ineficiencias podría encontrarse en la existencia de un diferencial entre tasas activas y tasas pasivas quizás excesivo y que genera ingresos netos disponibles demasiado altos. Existirian por lo menos dos vías posibles para resolver este problema; la primera sería una mayor competencia provocada por la creación y el desarrollo de canales alternativos de financiamiento y de intermediación financiera; la segunda sería una reducción en el encaje legal y un aumento de las tasas pasivas que force las tasas activas hacia la baja a través de una mayor disponibilidad de fondos prestables.

Antes de terminar este estudio, es necesario sin embargo enfati- 
zar el carácter muy tentativo de las conclusiones alcanzadas. Es claro que más investigación, a un nivel mucho más detallado, sería indispensable para poder validar las hipótesis expuestas anteriormente.

Método utilizado para el cál`ulo du ius, rticipaciones de ajuste

Se definen para cada una de las cuatro variables consideradas (ganancias, tasa activa, gastos en trabajo, gastos administrativos) valores de tendencia $\left\{\hat{Y}_{t}^{i}, i=1,4, t=72,79\right\}$ derivados por extrapolación logarítmica dè los valores históricos en el período 66-71. Se definen también para cada variable valores de ajuste $\left\{\bar{Y}_{\mathrm{t}}^{\mathrm{i}}, \mathrm{i}=1,4, \mathrm{t}=72,79\right\}$ obtenidos a partir del estado de resultados (1), sustituyendo los valores históricos por los valores de tendencia para las otras tres variables.

Para c ¿da año del período de crisis, se calcula entonces un valor de ajuste en la forma siguiente:

$$
Z_{t}^{i}=\left(Y_{t}^{i}-\hat{Y}_{t}^{i}\right) /\left(\bar{Y}_{t}^{i}-\hat{Y}_{t}^{i}\right) i=1,4, t=72,79
$$

en donde los $\mathrm{Y}^{\mathrm{i}}$ son valores históricos del período. Se calculan finalmente indices total de ajuste $\left\{\mathrm{g}^{\mathrm{i}}, \mathrm{i}=1,4\right\}$ como la suma ponderada de los ajustes anuales, $\mathrm{Z}_{\mathrm{t}}^{\mathrm{i}}$, el coeficiente de ponderación, $\mathrm{N}_{\mathrm{t}}^{\mathrm{i}}$, siendo proporcional a la participación del ajuste anual dentro del ajuste total:

$\mathrm{N}_{\mathrm{t}}^{\mathrm{i}}=\left|\overline{\mathrm{Y}}_{\mathrm{t}}^{\mathrm{i}}-\hat{\mathrm{Y}}_{\mathrm{t}}^{\mathrm{i}}\right| / \sum_{\mathrm{t}=2}^{\mathrm{\Sigma}} 7^{9}\left|\overline{\mathrm{Y}}_{\mathrm{t}}^{\mathrm{i}}-\hat{\mathrm{Y}}_{\mathrm{t}}^{\mathrm{i}}\right| \quad \mathrm{i}=1,4$

Los coeficientes totales de ajuste se obtienen entonces como:

$\mathrm{g}^{\mathrm{i}}=\sum_{\mathrm{t}=72}^{79} \mathrm{U}_{\mathrm{t}}^{\mathrm{i}} \mathrm{Z}_{\mathrm{t}}^{\mathrm{i}} \quad \mathrm{i}=1,4$

\section{BIBLIOGRAFIA}

Calderón, A. J., Cárdenas y A. Ize (1980), "Un análisis del mercado de crédito en México", Documento de trabajo. El Colegio de México.

Leibenstein H. (1976), A New Foundation for Microeconomics Harvard University Press. 